\title{
Robust Optimization of the Output Voltage of Nanogenerators by Statistical Design of Experiments
}

\author{
Jinhui Song ${ }^{1, \S}$, Huizhi Xie ${ }^{2, \S}$, Wenzhuo Wu', V. Roshan Joseph², C. F. Jeff Wü $(\bowtie)$, and Zhong Lin Wang ${ }^{1}(\bowtie)$ \\ ${ }^{1}$ School of Materials Science and Engineering, ${ }^{2}$ School of Industrial and Systems Engineering, Georgia Institute of Technology, Atlanta, \\ Georgia 30332-0205, USA
}

Received: 11 June 2010 / Accepted: 6 August 2010

(C) The Author(s) 2010. This article is published with open access at Springerlink.com

\begin{abstract}
Nanogenerators were first demonstrated by deflecting aligned $\mathrm{ZnO}$ nanowires using a conductive atomic force microscopy (AFM) tip. The output of a nanogenerator is affected by three parameters: tip normal force, tip scanning speed, and tip abrasion. In this work, systematic experimental studies have been carried out to examine the combined effects of these three parameters on the output, using statistical design of experiments. A statistical model has been built to analyze the data and predict the optimal parameter settings. For an AFM tip of cone angle $70^{\circ}$ coated with $\mathrm{Pt}$, and $\mathrm{ZnO}$ nanowires with a diameter of $50 \mathrm{~nm}$ and lengths of $600 \mathrm{~nm}$ to $1 \mu \mathrm{m}$, the optimized parameters for the nanogenerator were found to be a normal force of $137 \mathrm{nN}$ and scanning speed of $40 \mu \mathrm{m} / \mathrm{s}$, rather than the conventional settings of $120 \mathrm{nN}$ for the normal force and $30 \mu \mathrm{m} / \mathrm{s}$ for the scanning speed. A nanogenerator with the optimized settings has three times the average output voltage of one with the conventional settings.
\end{abstract}

\section{KEYWORDS}

$\mathrm{ZnO}$ nanowire arrays, robust parameter design, nanogenerator, piezoelectric properties

Nanogenerators, developed by Wang's group, constitute a new field in nanotechnology and energy harvesting with promising applications in building self-powered nanosystems [1-4]. The nanogenerator was discovered in 2006 by manipulating n-type $\mathrm{ZnO}$ nanowire arrays using a conductive atomic force microscope (AFM). Stimulated by this work [1], a prototype direct-current nanogenerator driven by ultrasonic waves without using an AFM was fabricated in 2007 [3]. Nanogenerators composed of single $\mathrm{ZnO}$ fine wire [5], nanowire and micro-fiber hybrid structure energy harvesting devices [6] and layer-by-layer stacked-up direct current generators [7] have all been demonstrated. Research on nanogenerators is now a new field in energy science [8]. A challenging issue affecting potential applications of nanogenerators is their low power output. In an attempt to obtain higher power output, p-type $\mathrm{ZnO}$ nanowire arrays, $\mathrm{ZnS}$ nanowires and other materials have all been used to fabricate nanogenerators [9-12], using a variety of different physical designs. However, a systematic study is still required in order to examine the influence of key parameters - such as the input mechanical force and electrode abrasion —on the output of a nanogenerator.

$\S$ These authors contributed equally to the work.

Address correspondence to Zhong Lin Wang, zhong.wang@mse.gatech.edu; C. F. Jeff Wu, jeffwu@isye.gatech.edu 
In this paper, we report a systematic experimental study of the effects of varying different parameters on the output of a nanogenerator, using our first nanogenerator based on conductive AFM scanning of n-type $\mathrm{ZnO}$ nanowire arrays. The experimental results were analyzed by statistical modeling, as a result of which the optimum choice of experimental parameters was identified. Experiments using the optimized parameters showed about a threefold enhancement in the output voltage of the nanogenerator in comparison to one with the conventional parameters.

The sample used in the experiments was aligned $\mathrm{ZnO}$ nanowire arrays grown on a GaN substrate by vapor deposition method [13]. Figure 1(a) shows a scanning electron microscopy (SEM) image of the $\mathrm{ZnO}$ nanowire arrays. A conductive AFM tip coated with $\mathrm{Pt}$ scanned over the $\mathrm{ZnO}$ nanowire array in contact mode. A $5 \times 10^{8} \Omega$ resistor was connected to the Pt-coated AFM tip and the sample substrate. When the tip scanned the sample, a closed-loop circuit is formed by the sample, tip, resistor and the sample, as indicated in the schematic illustration in Fig. 1(c). During the entire study the tip shape was kept unchanged, namely a Si tip of cone angle $70^{\circ}$ coated with Pt. The $\mathrm{ZnO}$ nanowires had diameters of $\sim 50 \mathrm{~nm}$ and lengths of $600 \mathrm{~nm}$ to $1 \mu \mathrm{m}$. The voltage across the resistor was monitored during the tip scan. No external voltage was applied during the entire scanning process. The voltage drop along the resistor comes from the deformation of the piezoelectric $\mathrm{ZnO}$ nanowires. Figure 1(b) shows a typical three-dimensional image of the electrical pulses generated by scanning over a $20 \mu \mathrm{m} \times 20 \mu \mathrm{m}$ area of aligned $\mathrm{ZnO}$ nanowires.

Normal force and scanning speed are two easily controllable parameters which can affect the output of the nanogenerator, as shown by previous experiments [10]. They can be tailored to optimize the output voltage. However, there are other uncontrollable parameters, which can vary over time and affect the performance of the nanogenerator. Such parameters
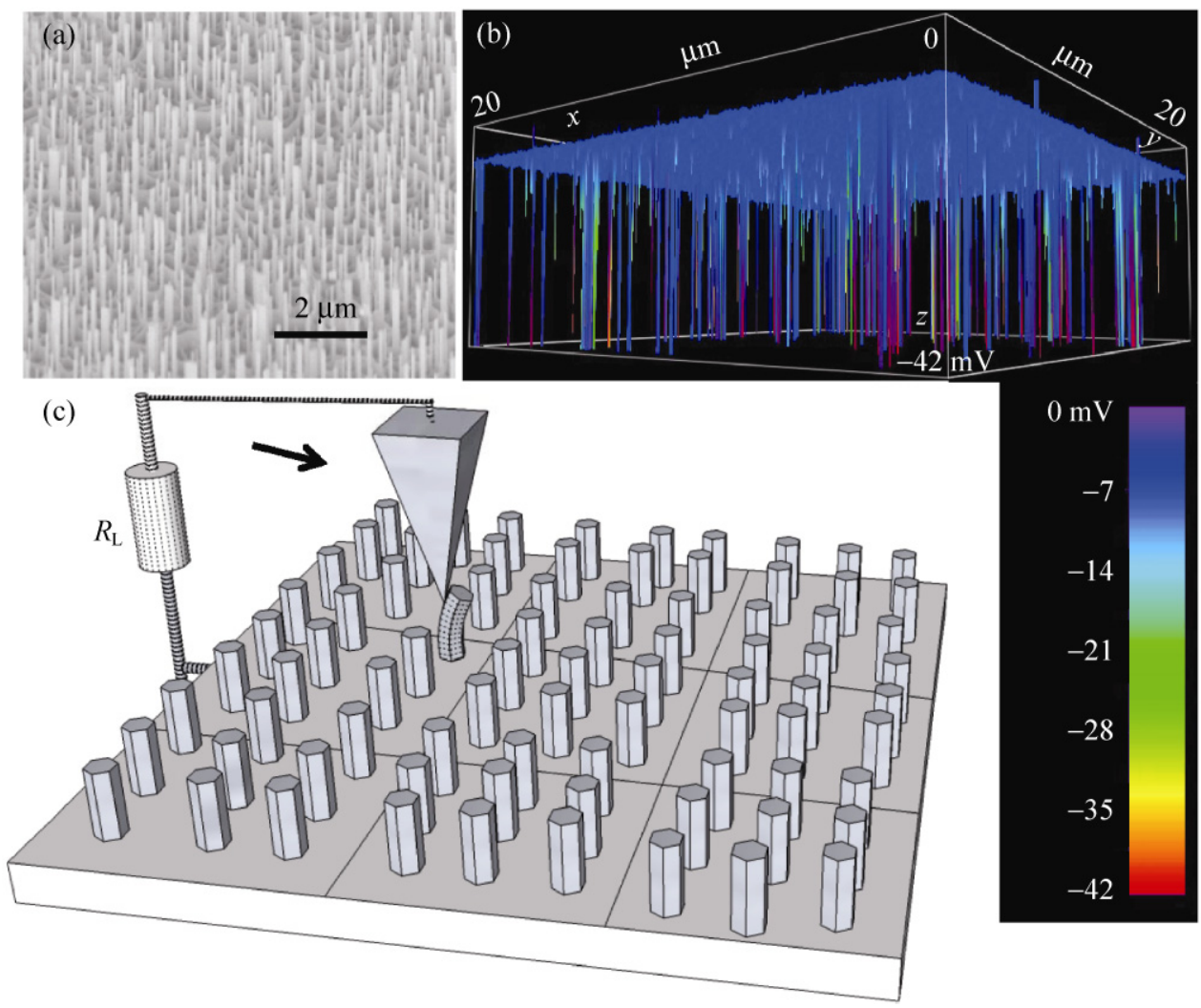

Figure 1 (a) SEM image of an aligned $\mathrm{ZnO}$ nanowire arrays grown on a GaN substrate. (b) Three-dimensional output of the electrical pulses generated by scanning an AFM tip across a $20 \mu \mathrm{m} \times 20 \mu \mathrm{m}$ area of an aligned ZnO nanowire array. (c) Schematic illustration of statistical design of experiments: a uniform $\mathrm{ZnO}$ nanowire array is divided into nine non-overlapping areas to make nine identical samples 
are called noise parameters in engineering statistics. Among the noise parameters, the tip abrasion seems to be the most critical one. The Pt coating can wear out over time due to the abrasive effect of the nanowires, leading to a reduction in the output voltage. An easy fix to this problem is to change the AFM tip frequently, but this would increase the cost. A technique commonly employed in industry to overcome this problem is robust parameter design [14]. The idea is to choose the controllable parameters so that the effect of the noise parameters on the output is minimized, thus making the product robust to the variations caused by the noise parameters.

Statistical design of experiments [14] was employed to identify the robust settings of the parameters. Three evenly-spaced levels of normal force and scanning speed were chosen, with the conventional setting (120 $\mathrm{mN}$ and $30 \mu \mathrm{m} / \mathrm{s}$, respectively) as the middle level. By choosing two levels, on either side of the conventional setting, we will be able to study the possible nonlinear effects of the parameters on the magnitude of the generated electrical pulses. The noise factor-tip abrasion-was studied by scanning the nanowires multiple times. The experimental design involved a three-level full factorial design on force and speed, which contains nine experimental runs [14]. In each run, we took 100 scans. Details of the design are shown in Table 1.

It is known that other factors like the quality and density of the nanowires can also affect the magnitude of the generated electrical pulse. To reduce the influence of these factors, the experiments were conducted by scanning different areas of the sample, i.e., the whole sample was divided into nine non-overlapping identical areas. Each area was then divided into two sub-areas. The nine different settings of normal forces and scanning speeds were randomly assigned to these nine areas. In each area, we began with a new AFM tip and randomly selected one of the two sub-areas. The AFM tip was then scanned 50 times over the nanowires in the sub-area with the assigned normal force and scanning speed. We then moved the AFM tip to the other sub-area within the same area and scanned it another 50 times. In this way we were able to study the effect of the abrasion of the AFM tip and also take into account the nanowire damage during the scanning process. The time order of the nine experiments was random. The experiments produced 900 three-dimensional images of electrical output like the one shown in Fig. 1(b). We calculated the total pulse generated for each image by taking the mean value of the magnitudes of all the pulses.

Figure 2 shows typical data from the experiments. These four plots come from four different areas among the nine sampled areas. In each plot, the blue dots represent the mean values of the electrical pulses from the first 50 scans, and the red stars represent the mean values from the next 50 scans. All the plots show a decreasing trend of the mean value of electrical pulses with respect to the number of scans. This indicates there is abrasion of the AFM tip because, as the number of scans increases, the AFM tip wears down resulting in lower output voltage. It is worth noting that the mean value of the electrical pulse decreases at different rates for different settings of the normal force and scanning speed. Furthermore, the

Table 1 Details of the statistical design of experiments

\begin{tabular}{|c|c|c|c|c|}
\hline \multirow{2}{*}{ Run } & \multirow{2}{*}{$\begin{array}{l}\text { Normal force } \\
\qquad(\mathrm{nN})\end{array}$} & \multirow{2}{*}{$\begin{array}{l}\text { Scanning speed } \\
(\mu \mathrm{m} / \mathrm{s})\end{array}$} & \multicolumn{2}{|c|}{ Area of sample } \\
\hline & & & 1 & 2 \\
\hline 1 & 86 & 10 & 1 st to 50 th scan & 51 st to 100 th scan \\
\hline 2 & 86 & 30 & 1 st to 50 th scan & 51 st to 100 th scan \\
\hline 3 & 86 & 50 & 1 st to 50 th scan & 51 st to 100 th scan \\
\hline 4 & 120 & 10 & 1 st to 50 th scan & 51 st to 100 th scan \\
\hline 5 & 120 & 30 & 1 st to 50 th scan & 51 st to 100 th scan \\
\hline 6 & 120 & 50 & 1 st to 50 th scan & 51 st to 100 th scan \\
\hline 7 & 154 & 10 & 1 st to 50 th scan & 51 st to 100 th scan \\
\hline 8 & 154 & 30 & 1 st to 50 th scan & 51 st to 100 th scan \\
\hline 9 & 154 & 50 & 1 st to 50 th scan & 51 st to 100 th scan \\
\hline
\end{tabular}




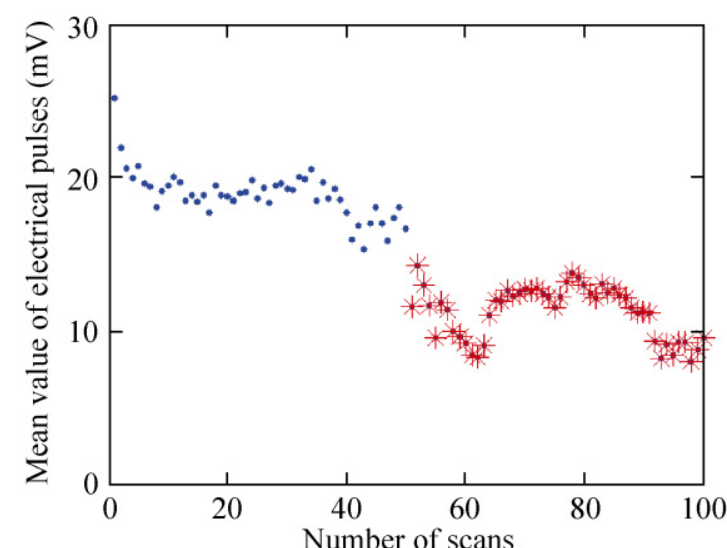

(a)

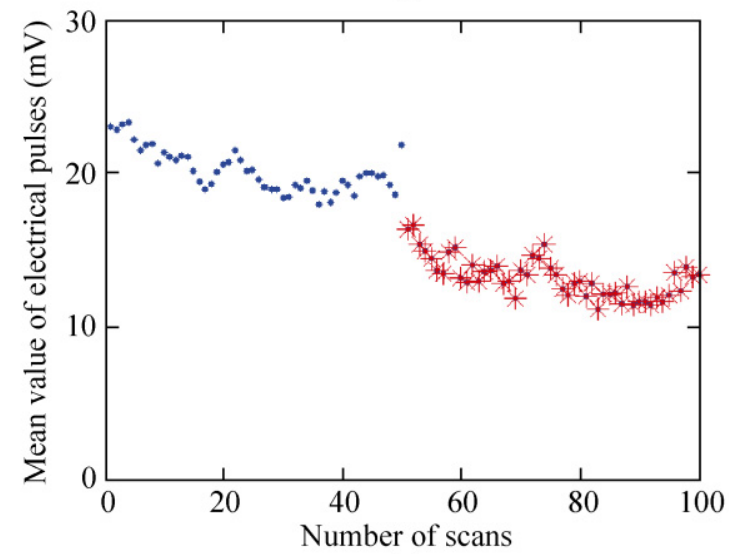

(c)

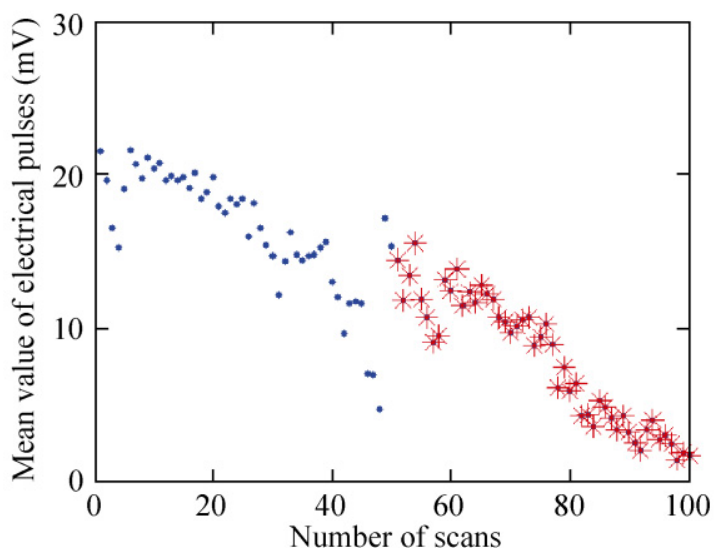

(b)

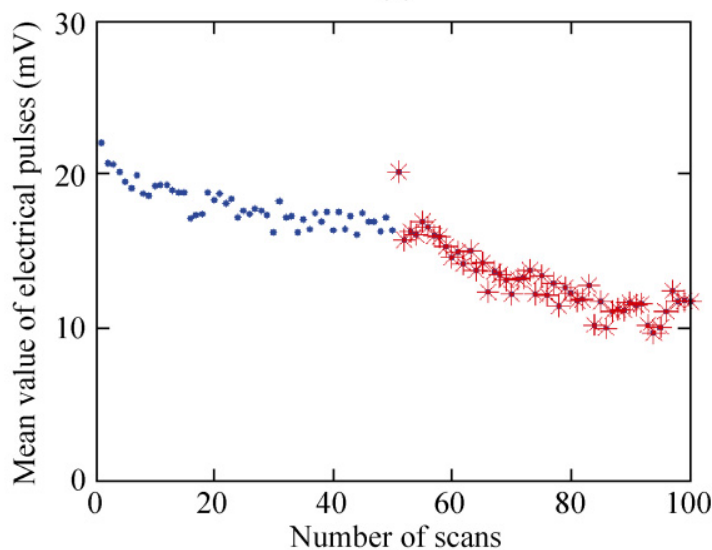

(d)

Figure 2 Scatter plot of the mean values of generated electrical pulses versus the number of scans for four settings of tip normal force and scanning speed. The blue dots represent the mean values of electrical pulses from the first 50 scans, and the red stars represent the mean values from the next 50 scans: (a) scatter plot for normal force $=120 \mathrm{nN}$, speed $=10 \mu \mathrm{m} / \mathrm{s}$; (b) scatter plot for force $=120 \mathrm{nN}$, speed $=30 \mu \mathrm{m} / \mathrm{s}$; (c) scatter plot for force $=154 \mathrm{nN}$, speed $=30 \mu \mathrm{m} / \mathrm{s}$; (d) Scatter plot for force $=86 \mathrm{nN}$, speed $=50 \mu \mathrm{m} / \mathrm{s}$

mean values of the electrical pulse generated in the first scan of each area, when the AFM tip is new, are different for different settings of normal force and scanning speed (see Fig. 2).

The main effect plots [14] for force and speed are shown in Fig. 3. We can see that both force and speed have significant effects on the output voltage. We cannot choose the optimum settings of the force and speed using these plots, because our objective is not only to maximize the output voltage but also to identify their robust settings in order to minimize the effect of tip abrasion on output. For this purpose, we fit the data to the following statistical model:

$$
y_{n}=f_{1} \exp [-r(n-1)]+\varepsilon
$$

where $n$ is the number of scans, $y_{n}$ is the mean value of the electrical pulse at the $n$th scan, $f_{1}$ is the mean value of the electrical pulses in the first scan, $r$ is a non-negative constant that captures the effect of tip wear rate (on the logarithmic scale), and $\varepsilon$ is the random error. A nanogenerator will produce high output voltage throughout its usage if the mean value of the generated electrical pulse in the first scan is large and if the wear rate is small. Thus, the objective is to find a setting of normal force and scanning speed so that $f_{1}$ is as large as possible and $r$ as small as possible. By reducing $r$, we can make the performance of a nanogenerator less susceptible to tip abrasion. We fitted the model in Eq. (1) using nonlinear least squares separately for each area [15]. The estimates of $f_{1}$ and $r$ for different normal forces and scanning speeds are displayed as contour plots in Fig. 4, which shows that 


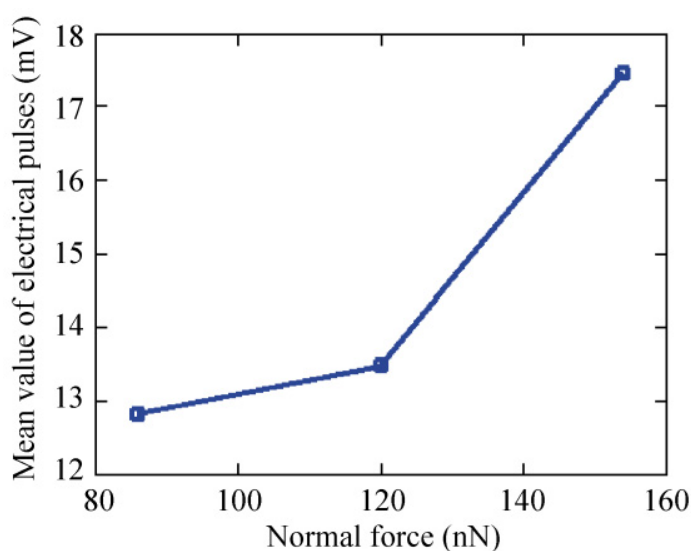

(a)

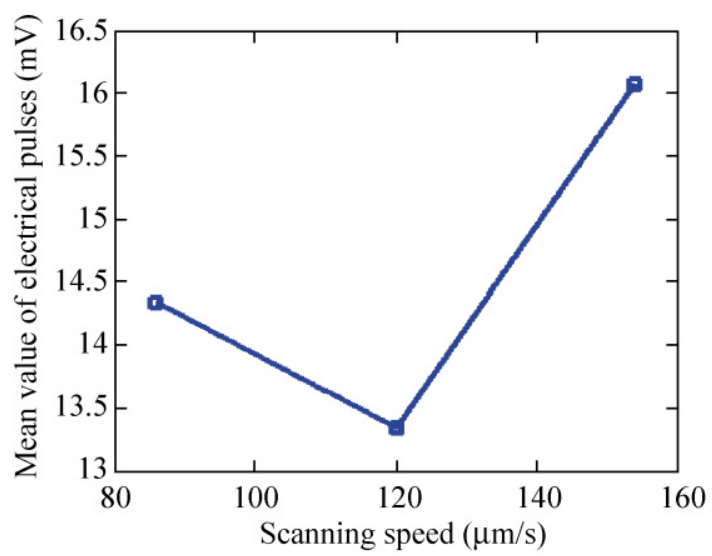

(b)

Figure 3 Main effect plots of (a) normal force and (b) scanning speed

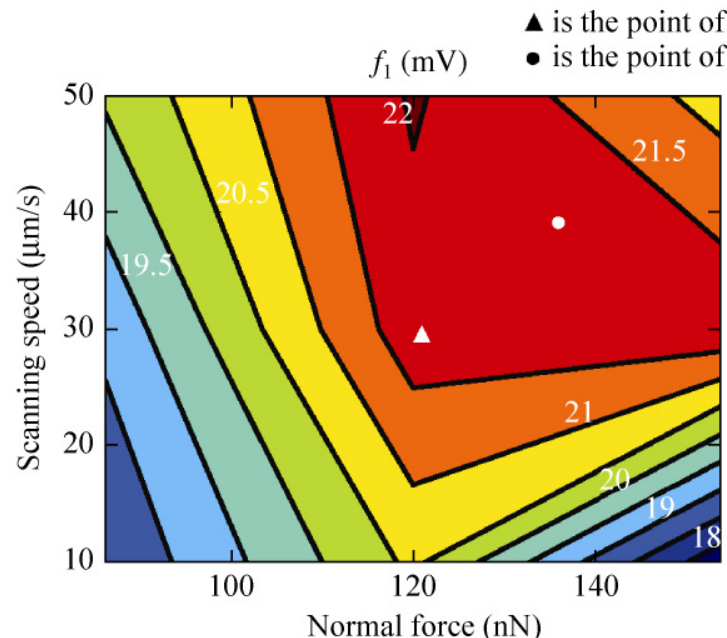

(a)

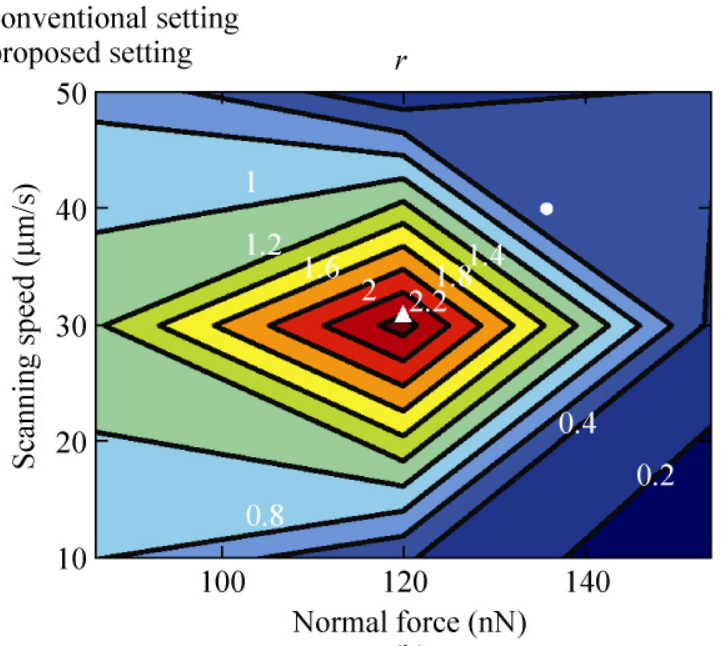

(b)

Figure 4 Contour plots of (a) the estimated values of $f_{1}$ and (b) $r$ for different normal forces and scanning speeds

the usual setting of a normal force of $120 \mathrm{nN}$ and scanning speed of $30 \mu \mathrm{m} / \mathrm{s}$ is not optimum because it leads to a rapid rate of decrease $r$ in spite of the high mean value of the electrical pulse in the first scan $f_{1}$. Therefore we propose to use a new setting comprising a normal force of $137 \mathrm{nN}$ and scanning speed of $40 \mu \mathrm{m} / \mathrm{s}$. This gives a mean value of electrical pulse in the first scan $f_{1}$ as high as that for the conventional setting, yet a much lower rate of decrease $r$.

A possible physical explanation for this interesting phenomenon is as follows. The $\mathrm{ZnO}$ nanowires cannot be bent to a sufficiently large angle with too small a normal force. A larger angle leads to a higher strain, which results in a stronger piezoelectric field. On the other hand, too high a normal force can chop off the nanowires very quickly and cause a dramatic decrease in generated electrical pulse after a few scans. Our proposed setting of the normal force is large enough to bend the nanowires to a large angle, but not sufficiently large to chop them off. For a fixed normal force, higher scanning speed can reduce the charge leakage time, which results in higher output. However too high a scanning speed will increase the chance of an AFM tip losing contact with the compressed side of the nanowires. Our proposed setting of the scanning speed might be just at the point where the leakage time is very small and yet the tip does not easily lose contact with the compressed side of the nanowires. Since no good physical model is available to explain such subtle differences, an empirical approach based

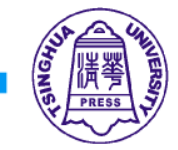


on statistical design of experiments is an effective way to tackle this problem.

With the proposed new settings, we carried out two sets of confirmatory experiments. We randomly chose two areas from a newly grown nanowire sample. The proposed setting $(137 \mathrm{nN}, 40 \mu \mathrm{m} / \mathrm{s})$ and the conventional setting $(120 \mathrm{nN}, 30 \mu \mathrm{m} / \mathrm{s})$ were randomly assigned to the two areas. In each area, a new AFM tip was scanned 50 times across the nanowires with the assigned setting. The outputs are shown in Fig. 5. It is clearly seen that the mean value of the electrical pulses for the proposed new setting is stable and shows much less fluctuation than that for the conventional setting, while the mean values of electrical pulse in the first scan are almost the same for the two settings. Thus we have achieved the robustness objective, i.e., as the tip ages, the pulse decreases much more slowly for the new setting than for the conventional setting. The average of the 50 mean values of the electrical pulses using the proposed new setting is almost three times that obtained with the conventional setting parameters.

In summary, we have systematically investigated the effects of normal force, scanning speed, and abrasion of the AFM tip on the magnitude of the electrical pulse produced by a nanowire nanogenerator. A statistical model has been proposed to quantify the

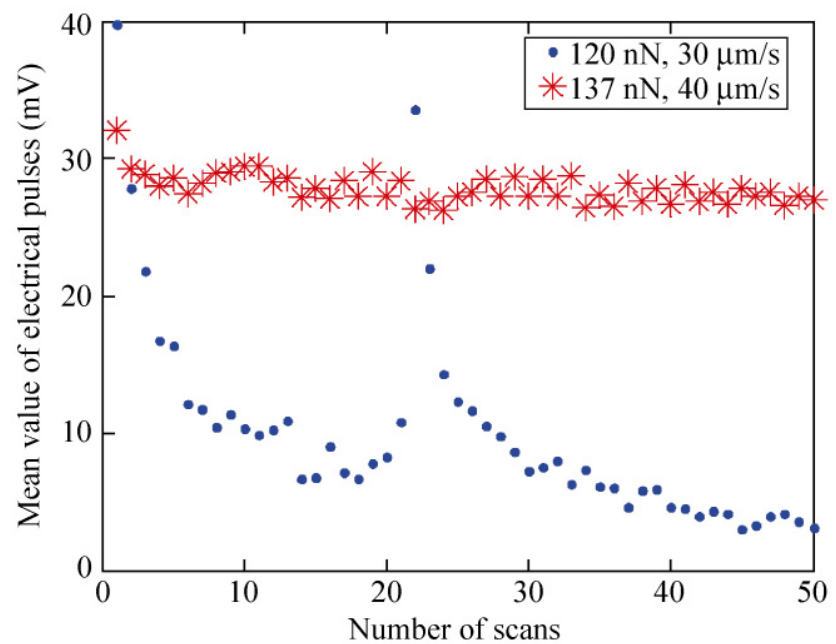

Figure 5 Comparison of the output voltage obtained using the proposed new setting and the conventional setting. The red stars are the mean values of electrical pulses for the proposed new setting. The blue dots are the mean values of electrical pulses for the conventional setting. The average of the 50 mean values of electrical pulses with the proposed new setting is almost three times that obtained with the conventional setting effects of varying these parameters. A new setting of normal force and scanning speed was found to give improved magnitudes of the generated electrical pulses. The new setting produces higher output mainly because the output is more robust to the abrasion of the AFM tip. Confirmatory experiments showed that the proposed setting does indeed significantly improve the magnitude of the generated electrical pulse. This work shows the possibility of applying statistical design of experiments and robust parameter design in optimizing the performance of nanodevices for practical applications.

\section{Acknowledgements}

This research was supported by the Basic Energy Science, U.S. Department of Energy (BES DOE) (No. DE-FG02-07ER46394), the National Science Foundation (NSF) (Nos. DMS0706436, CMMI 0403671), and the King Abdullah University of Science and Technology (KAUST) Global Research Partnership.

Open Access: This article is distributed under the terms of the Creative Commons Attribution Noncommercial License which permits any noncommercial use, distribution, and reproduction in any medium, provided the original author(s) and source are credited.

\section{References}

[1] Wang, Z. L.; Song, J. H. Piezoelectric nanogenerators based on zinc oxide nanowire arrays. Science 2006, 312, 242-246.

[2] Song, J. H.; Zhou, J.; Wang, Z. L. Piezoelectric and semiconducting coupled power generating process of a single $\mathrm{ZnO}$ belt/wire. A technology for harvesting electricity from the environment. Nano Lett. 2006, 6, 1656-1662.

[3] Wang, X. D.; Song, J. H.; Liu, J.; Wang, Z. L. Directcurrent nanogenerator driven by ultrasonic waves. Science 2007, 316, 102-105.

[4] Xu, S.; Qin, Y.; Xu, C.; Wei, Y. G.; Yang, R. S.; Wang, Z. L. Self-powered nanowire devices. Nat. Nanotechnol. 2010, 5, 366-373.

[5] Yang, R. S.; Qin, Y.; Dai, L. M.; Wang, Z. L. Power generation with laterally-packaged piezoelectric fine wires. Nat. Nanotechnol. 2009, 4, 34-39.

[6] Qin, Y.; Wang, X. D.; Wang, Z. L. Microfibre-nanowire 
hybrid structure for energy scavenging. Nature 2008, 451, 809-813.

[7] Xu, S.; Wei, Y. G.; Liu, J.; Yang, R.; Wang, Z. L. Integrated multilayer nanogenerator fabricated using paired nanotipto-nanowire brushes. Nano Lett. 2008, 8, 4027-4032.

[8] Service, R. F. Nanogenerators tap waste energy to power ultrasmall electronics. Science 2010, 328, 304-305.

[9] Lu, M. Y.; Song, J. H.; Lu, M. P.; Lee, C. Y.; Chen, L. J.; Wang, Z. L. ZnO-ZnS heterojunction and $\mathrm{ZnS}$ nanowire arrays for electricity generation. ACS Nano 2009, 3, 357-362.

[10] Lu, M. P.; Song, J. H.; Lu, M. Y.; Chen, M. T.; Gao, Y. F.; Chen, L. J.; Wang, Z. L. Piezoelectric nanogenerator using p-type ZnO nanowire arrays. Nano Lett. 2009, 9, 1223-1227.
[11] Wang, X. B.; Song, J. H.; Zhang, F.; He, C. Y.; Hu, Z.; Wang, Z. L. Electricity generation based on one-dimensional group-III nitride nanomaterials. Adv. Mater. 2010, 22, 2155-2158.

[12] Huang, C. T.; Song, J. H.; Lee, W. F.; Ding, Y.; Gao, Z. Y.; Hao, Y.; Chen, L. J.; Wang, Z. L. GaN nanowire arrays for high-output nanogenerators. J. Am. Chem. Soc. 2010, 132, 4766-4771.

[13] Pan, Z. W.; Dai, Z. R.; Wang, Z. L. Nanobelts of semiconducting oxides. Science 2001, 291, 1947-1949.

[14] Wu, C. F. J.; Hamada, M. S. Experiments: Planning, Analysis and Optimization, 2nd ed.; Wiley: New York, 2009.

[15] Draper, N. R.; Smith, H. Applied Regression Analysis, 3rd ed.; Wiley: New York, 1998. 